\title{
Update on medical management of dyslipidemia and atherosclerosis
}

\author{
Ginter $E^{1}$, Simko $V^{2}$ \\ Institute of Preventive and Clinical Medicine, Bratislava, Slovakia.ginter.emil@mail.t-com.sk
}

\begin{abstract}
Scientific achievements revealing the pathogenesis of atherosclerosis resulted in the second half of the 20th century in major improvement in prevention and therapy of cardiovascular disorders (CVD). Essential became the understanding of a critical pathogenetic role of the low-density lipoproteins (LDL), mainly their oxidized form (oxLDL) and also the protective potential of the high-density lipoproteins (HDL). CVD is now regarded to be an inflammatory disease in which a systemic inflammatory reaction is combined with an accumulation of immune cells in atherosclerotic plaques. Higher intake of antioxidants in fruit and vegetable, life style modifications, cessation of smoking, physical exercise and introduction of medications that lower LDL and promote HDL (statins, niacin and fibrates) resulted in a substantial decline of the killer effect of unmanaged CVD. In the United Kingdom the male CVD mortality declined between 1970 and 2009 from 700 to 200 deaths per 100,000. In France, CVD mortality in the middle age population (25-64 years) is now responsible for death in only $15 \%$ men and in $11 \%$ women. Unfortunately, in many parts of the world CVD mortality remains a prominent population scourge. Recent dicoveries, especially on the role of peroxisome proliferator-activated receptors (PPAR) and antisense compounds used in addition to established anti-atherogenic medications, promise further gains in the fight against atherosclerosis (Fig. 4, Ref. 54). Full Text in PDF www.elis.sk.

Key words: cardiovascular disease, LDL, ox-LDL, HDL, statins, niacin, fibrates, peroxisome proliferator, antisense compounds.
\end{abstract}

Arterial stiffening is a complex process involving extracellular matrix proteins and smooth muscle cells $(1,2)$. Increased arterial stiffness generates CVD. Although the exact mechanisms underlying arterial stiffening are not well understood, oxidative stress, inflammation and immunological factors play an important role. CVD is an inflammatory disease in which a systemic inflammatory reaction is combined with an accumulation of immune cells, such as monocytes/macrophages, dendritic cells and numerous lymphocytes, in atherosclerotic plaques. The oxidative transformation of low-density lipoprotein (LDL) is recognized as a key step in the initiation and progression of atherosclerosis. Oxidized-LDL are associated with stimulation of collagen synthesis in arterial smooth muscle cells, they promote intimal thickening and impair nitric oxide bioactivity, all of which may contribute to arterial stiffness. Thus ox-LDL and inflammation are the driving forces in atherosclerosis (3). Strong evidence supports the central role of proinflammatory cytokines, such as interleukin-1 $\beta$ (IL$1 \beta$ ) and interleukin-6 (IL-6) in CVD (4). The role of peroxisome proliferator-activated receptors (PPAR) in CVD is now receiving widespread attention. As ligand-activated nuclear receptors, they play a role in regulation of lipid metabolism. This feature of the

${ }^{1}$ Institute of Preventive and Clinical Medicine, Bratislava, Slovakia (emeritus), and ${ }^{2}$ State University of New York, Downstate Medical Center at Brooklyn, USA

Address for correspondence: E. Ginter, RND, DSc, Racianska 17, SK83102 Bratislava, Slovakia.
PPARs has been successfully exploited to manage the metabolic syndrome, associated with hypertension, hyperlipidemia and CVD (5). Thrombosis, the formation of a blood clot within the blood vessel resulting in occlusion of blood flow, is a major problem that accompanies CVD. The therapeutic targeting of platelets is recognized as effective in the prevention and treatment of CVD (6, 7). New cholesterol absorption inhibitors were found. Ezetimibe initially raised hopes because it blocks the absorption of dietary and biliary cholesterol, resulting in intracellular cholesterol depletion (8). The firmly established benefit of statins in lowering the LDL has been further enhanced by combining statins with niacin and fibrates. Novel discoveries leading to a combination of antisense technology with PPARs, may represent a further important breakthrough in the management of dyslipidemia.

\section{Established medications used in lipid disorders}

Statins

Presently, statins represent the main agent in combating atherosclerosis (9). Numerous epidemiologic and angiographic studies indicate that elevated serum LDL is a major cause of CVD. Statins act by inhibiting HMG-CoA reductase, the rate limiting enzyme for cholesterol synthesis. By decreasing elevated serum LDL-cholesterol concentration statins can reduce the incidence and progression of CVD (10-12). Although manufacturer brand names may vary by regions, the main representantives of statins are atorvastatin $\left(\right.$ Lipitor $\left.{ }^{\circledR}\right)$, simvastatin $\left(\right.$ Zocor $\left.{ }^{\circledR}\right)$, pravastatin 
(Pravachol $\left.{ }^{\circledR}\right)$, lovastatin $\left(\right.$ Mevacor ${ }^{\circledR}$, Altocor $\left.{ }^{\circledR}\right)$, fluvastatin (Lescol $\left.{ }^{\circledR}\right)$ and rosuvastatin $\left(\right.$ Crestor $\left.{ }^{\circledR}\right)$. Another candidate, cerivastatin was withdrawn for inducing more pronounced muscle disorders and even rhabdomyolysis, compared with other statins. There is another new statin, Pitavastatin (Livalo) (13).

It was found that there is a residual cardiovascular risk after treatment with statins. This residual risk is partially due to low HDL-C and high triglycerides (TG), despite achieving LDL lowering with statins. Livalo, approved on the basis of 10 clinical trials comparing efficacy and safety with atorvastatin, simvastatin and pravastatin, offered LDL-C lowering of up to $45 \%$ at a $4 \mathrm{mg}$ dose, at the same time decreasing TG and elevating HDL-C (13). Fixed combinations of statins with other drugs are also available: Simcor is simvastatin wit extended release niacin, advicor is lovastatin with niacin.

\section{Nicotinic acid and niacin}

Niacin is a derivative of nicotinic acid used for management of dyslipidemia for several past decades. It is a water-soluble part of vitamin B complex (also known as vitamin B3 or vitamin PP). Niacin has anti-hyperlipidemic effects: In pharmacological doses it reverses atherosclerosis by reducing total cholesterol, TG, verylow-density lipoprotein (VLDL), LDL, and it increases HDL. It has been proposed that niacin has the ability to lower lipoprotein (a) (14). In general, niacin decreases LDL-C by about $5 \%$ to 25 $\%$, increases HDL-C by $15 \%$ to $35 \%$, and decreases TG by 20 $\%$ to $50 \%$.

Potential residual cardiovascular risk after treatment with statins due to low HDL-C and high TG, despite lowering of LDL may be resolved by combining statins with niacin (15). Flushing is an unpleasant side effect of niacin therapy. A new formulation of niacin shows a slower rate of absorption, substantially reducing flushing, when compared with the traditional niacin (16). Considering beneficial effect of medications on lipid metabolism, new stricter criteria also focus on the effect on sclerotic arterial wall thickness. Efficiency of niacin regarding its effect on arterial wall has been questioned and there have been suggestions to use niacin only as monotherapy, as a last resort. With the recent discovery of a specific receptor for nicotinic acid (GPR109A) (17), the molecular mechanisms underlying the pharmacological effects of nicotinic acid have become clearer. The receptor is expressed in brown and white adipose tissue, in various immune cells, including monocytes, macrophages, dendritic cells and neutrophils.

GPR109A is coupled to Gi type G proteins. Its activation by nicotinic acid results in a Gi-mediated inhibition of adenylyl cyclase, resulting in a decrease in intracellular cyclic AMP, the principal mediator of adipocyte lipolysis. Strong evidence exists that at least the initial steps of the nicotinic acid-induced changes in lipid metabolism are mediated by GPR109A (18). New agents acting via the nicotinic acid receptor are currently being developed.

\section{Fibrates}

These belong to a class of amphipathic carboxylic acids that exert an impressive therapeutic effect when combined with statins.
Basic mechanism of action of peroxisome proliferator-activated receptors (PPARs) and effects on gene expression

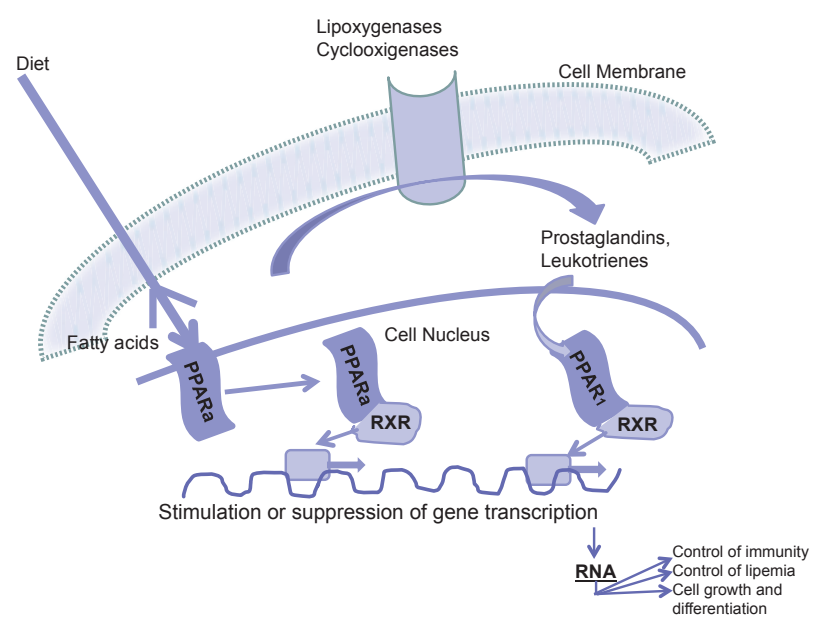

Fig. 1. Peroxisome proliferator-activated receptors (PPARs) are a group of nuclear receptor proteins that function as transcription factors regulating the expression of genes. All PPARs heterodimerize with the retinoid $X$ receptor (RXR) and bind to specific regions on the DNA of target genes. Partly according to Wikipedia.

Commonly presribed are: Bezafibrate (e.g. Bezalip), Ciprofibrate (e.g. Modalim), Gemfibrozil (e.g. Lopid) and Fenofibrate (e.g. TriCor). Originally frequently prescribed Clofibrate (Atromid), the „grandfather" of fibrates, fell in disfavor for its side effects, among other for potentially promoting gallstones. Fibrates decrease tissue cholesterol by promoting cholesterol excretion to the bile. Following long term use, bile supersaturates with cholesterol, resulting in gallstone formation. However, in none of the extensive clinical trials with Gemfibrozil and Fenofibrate there was an evidence for increase in the incidence of gallstones.

Fibrates may be used as monotherapy, reducing the incidence of non fatal coronary vascular accidents. However, used alone they were not found to improve all cause mortality. As monotherapy, fibrates are indicated only in patients intolerant to statins.

A well known paper originating from Helsinki (19) documented that Gemfibrozil caused a marked increase in HDL and persistent reductions in serum levels of LDL and TG. More recently evidence was reported that fenofibrate added to a statin was beneficial in patients with diabetes mellitus who had acceptable LDL levels, but persistent, significant hypertriglyceridemia and low HDL (20). Fibrates are structurally related to thiazolidinediones, a novel class of antidiabetics that also influence PPRs.

In the 1990s it was revealed that fibrates activate the peroxisome proliferator-activated receptors (PPARs). These represent a group of nuclear receptor proteins that function as transcription factors regulating the expression of genes. PPARs play essential roles in the regulation of cellular differentiation, development, and lipid metabolism (Fig. 1). Three types of PPARs have been identified: alpha, gamma, and delta (beta): $\alpha$ (alpha) - expressed in liver, kidney, heart, muscle, adipose tissue, $\beta / \delta$ (beta/delta) expressed in many tissues but markedly in brain, adipose tissue, 
and skin; $\gamma$ (gamma) - expressed in virtually all tissues, including heart, muscle, colon, kidney, pancreas, spleen, in macrophages, large intestine and white adipose tissue.

All PPARs heterodimerize with the retinoid X receptor (RXR) and bind to specific regions on the DNA of target genes. Endogenous ligands for the PPARs include free fatty acids, prostaglandins and leukotrienes that originate from free fatty acids under the effect of lipooxygenase and cyclooxygenase. PPAR $\alpha$ and PPAR $\gamma$ are the molecular targets of a number of marketed drugs. Fibrates activate PPAR- $\alpha$ receptor in muscle, liver, and other tissues. The mechanism underlying the beneficial effect of PPARs is not confined to whole body metabolism, but also includes modulation of other vital processes, such as inflammation and cell fate (proliferation, differentiation, apoptosis). PPARs are presumed therapeutic targets in CVD (21). A word of caution: at present the clinical trials have not produced convincing evidence that CVD is prevented with the use of PPARalpha and PPARgamma agonists (22).

\section{Antioxidants}

Free oxygen radicals represent potential risk for the arterial vasculature by stimulating the rise of ox-LDL. Antioxidants terminate these reactions by removing free radical intermediates and inhibit other oxidation reactions. Essential natural antioxidants in the diet are ascorbic acid (vitamin C), $\alpha$-tocopherol (vitamin E), carotenes and polyphenols (PPH) (e.g. resveratrol). Additional antioxidants are represented by the glutathione, lipoic acid and ubiquinol (coenzyme Q).

Polyphenols are a class of chemical compounds consisting of a hydroxyl group $(-\mathrm{OH})$ bonded directly to an aromatic hydrocarbon group. Phenol forms a redox system with chinone and therefore dietary PPH (for example skin of red grapes) can directly scavenge free oxygen radicals and downregulate expression of proinflammatory mediators, adhesion molecules, and growth factor receptor genes. These polyphenolic compounds have potential therapeutic value as antioxidant and anti-inflammatory agents in the management of CVD. Much attention has been recently focused on resveratrol. This compound was reported in experimental animals and in short term human studies $(23,24)$ to demonstrate cardioprotective effects. Moore human studies are needed before resveratrol is considered a standard therapeutic agent.

Vitamin E: Several in vitro and animal studies, observational as well as clinical trials suggested a cardioprotective effect of tocopherol (25). It increases oxidative resistance in vitro and prevents atherosclerotic plaque formation in mouse models. However, this remains controversial since clinical studies have not demonstrated a benefit of vitamin $\mathrm{E}$ in the primary and secondary prevention of cardiovascular disease. Nearly 200 trials using vitamin E supplements were carried out in the decade after the initial report, then followed by a recent review and meta-analysis. Their concludsion: vitamin E represents neither benefit nor harm (26). The American Heart Association does not support the use of vitamin E supplements to prevent cardiovascular disease, but does recommend the consumption of foods abundant in antioxidant vitamins.

Carotenoids are a class of natural fat-soluble pigments found in plants. The most abundant carotenoids in the diet are beta-carotene, lycopene, lutein, beta-cryptoxanthin, zeaxanthin and astaxanthin. Numerous epidemiologic studies have supported the hypothesis that their antioxidative action could be used as an inexpensive means of prevention and possibly treatment of cardiovascular diseases (27). More recently, sobering conclusions were reported from a multicenter, randomized, double-blind, placebo-controlled primary prevention trial, involving smokers, former smokers and workers exposed to asbestos. When the effects of a combination of beta carotene and vitamin A on the incidence of lung cancer and CVD were compared with those of placebo, the outcome was astounding: mortality related to lung cancer and CVD in the treatment groups was actually higher than on placebo. On the basis of these findings, the randomized trial was stopped 21 months earlier than planned (28). There is a speculation that the high intake of beta carotene competitively inhibited absorption of lycopene, an oxygenated carotenoid with great antioxidant properties. Lycopene has antiatherogenic effect in experimental animals (29).

Vitamin $C$ is a pivotal redox modulator in many biological reactions. Growing epidemiological, clinical and experimental evidence now suggests a more specific role of ascorbate in vasomotion and in the prevention of atherosclerosis (30). For endothelial cells, ascorbate helps to prevent endothelial dysfunction, stimulates type IV collagen synthesis, and enhances cell proliferation. For vascular smooth muscle cells, ascorbate inhibits dedifferentiation, recruitment, and proliferation in areas of vascular damage. For macrophages, ascorbate decreases oxidant stress related to their activation, decreases uptake and degradation of ox-LDL (31). Long term deficiency of ascorbate promotes atherosclerosis-like injury in experimental animals. It is possible that its deficiency promotes human atherosclerosis as well (32). There is a sparsity of randomized, double-blind, placebo-controlled prevention trials to document the role of chronic vitamin $\mathrm{C}$ deficiency in human atherogenesis.

In summary, despite the lack of strong evidence for antioxidants to prevent CVD, it is reasonable to assume their beneficial potential. Plant food is the main source of antioxidants. What is the evidence? Meta-analysis of several prospective studies showed no significant difference in the mortality caused by various types of cancer and stroke between vegetarians and "health-conscious" nonvegetarians. The effect of predominantly plant food is complex. In vegetarians a decrease in ischemic heart disease mortality was observed due to lower cholesterol levels, lower prevalence of obesity, non-smoking and higher consumption of antioxidants (33). In the near future we expect a very large cohort study of diet and health on more than half million of persons, the European Prospective Investigation into Cancer and Nutrition (EPIC) study, to bring new data on the relationships between diet, lifestyle, environmental factors and the incidence of cancer, CVD and other chronic diseases. Preliminary results suggest that a higher intake of fruits and vegetables is associated with a reduced risk of CVD mortality (34).

\section{Long-chain $n$-3 fatty acids}

A type of fatty acids, popularly referred to as omega-3 fatty acids are a family of essential polyunsaturated fatty acids that have 
in common a final carbon-carbon double bond in the $n-3$ position; that is, the third bond from the methyl end of the fatty acid (n-3). Nutritionally important long-chain $n-3$ fatty acids include eicosapentaenoic acid (EPA), and docosahexaenoic acid (DHA). Interest in $\mathrm{n}-3$ was initiated by observing that the Inuits (Eskimos) living in the Arctic, have very low CVD mortality while consuming practically no plant food $(35,36)$. However, they consume high amounts of $n-3$ from fatty fish and have increased HDL cholesterol and low TG.

The association between high intake of $n-3$ and decreased morbidity and mortality from CVD can be explained by several mechanisms: $n-3$ reduce the synthesis of VLDL particles and increase TG removal from VLDL through the upregulation of the lipoprotein lipase (37). Inhibitory effect on atherothrombosis by n-3 includes the modulation of the expression of pro-atherogenic genes (e.g. endothelial leukocyte adhesion molecules, inflammatory cytokines and cyclooxygenase). Positive effect of n-3 on cardiac arrhythmia includes complex interactions with ion channels (sodium, potassium and calcium channels), typically requiring the presence of free n-3 (38). N-3 increase the production of bioactive lipid mediators (protectins and resolvins) affecting cytokine-induced signal transduction. Mechanisms of beneficial effet of $n-3$ suggest a synergic action of $n-3$ with statins. COMBOS study (Combination of Prescription Omega-3 With Simvastatin) achieved significant improvements across a range of lipid indicators beyond the LDL primary target, including TG and lipoprotein particle size (39).

\section{Bile acid sequestrants (BAS)}

BAS were among the first drugs approved to lower cholesterol. BAS are positively charged resins that bind to negatively-charged bile acids in the intestine and prevent their absorption. To compensate for the loss of bile acids, the liver increases the conversion of cholesterol to bile acids. The conversion of cholesterol to bile acids reduces cholesterol in the body, resulting in a drop of cholesterol levels in the blood. However, the BAS might also increase the production of TG. BAS cause a small increase of HDL. However, when combined with statins, the BAS can provide an additional reduction in LDL. Some of the side effects of BAS are constipation, flatulence, indigestion, GI distress, decreased absorption of many drugs such as statins, niacin, levothyroxine, and digoxin. Presently, BAS are seldom used. Instead, intestinal absorption of cholesterol may be controlled by Ezetimibe, a selective inhibitor of intestinal cholesterol absorption (40). Initial enthusiasm for Ezetimibe was tempered after a trial comparing extended release niacin with Ezetimibe (41). While niacin brought HDL up, Ezetimibe may actually lower HDL and when it is used alone there is a risk of atherogenicity.

\section{Novel antiatherogenic medications}

Numerous compounds already in use modulate lipid-lowering effect of statins. The dominant therapeutic role of statins is being threatened for inadequate suppression of TG and weak effect on HDL. For antilipid therapy to be fully effective, in some patients statins have to be combined with supplementary medications.
Because various agents, fibrates, niacin, ezetimibe, phytosterols, omega-3 fatty acids and statins each regulate serum lipids by different mechanisms, combination therapy could be more helpful in achieving a comprehensive lipid control when compared with statin monotherapy (42-44).

To promote statin effectiveness there has been a sequence of candidate pharmaceuticals and biochemical agents. These include new and interesting therapeutic options, for example rate-limiting proteins (including Niemann-Pick C1-like 1 (NPC1L1), microsomal TG transfer protein (MTP), acyl-coenzyme A/cholesterol acyltransferase (ACAT), acyl CoA: diacylglycerol acyltransferases 2 (DGAT2), proprotein convertase subtilisin/kexin type 9 (PCSK9)), nuclear receptors (farnesoid X receptor (FXR), thyroid hormone receptor (TR) (45). Novel pharmacological targets for raising HDL-C also include the nuclear liver $\mathrm{X}$ and farnesoid $\mathrm{X}$ receptors and endothelial lipase (46). Traditional Chinese medicine recommends statin supplementation with Zhibitai derived from extracts of red yeast rice, Chinese hawthorn, oriental water plantain rhizome and large head atractylodes rhizome (47).

The secretory phospholipase A2 enzyme (sPL A2) inhibitor A-002 could be an effective anti-athersclerotic agent (48). To improve the efficacy of current anti-platelet therapies, a small number of characterized platelet receptors which include proven anti-platelet drug targets, are currently the focus of drug discovery and clinical trials for the prevention and treatment of thrombosis. These include ADP receptor $\left(\mathrm{P} 2 \mathrm{Y}_{12}\right)$ antagonists and molecules that inhibit activation of PARs for thrombin (49). Novel research on statin pharmacology revealed that the pleiotropic effects of statins are associated with anti-inflammatory activity, enhanced endothelial function and inhibition of oxidative stress (50).

\section{Antisense technology}

Anisense technology is a new direction in discovering more effective therapies. When the genetic sequence of a particular gene is known to be causative of a particular disease, it is possible to synthesize a strand of nucleic acid that will bind to the messenger

Antisense DNA strands interfere with protein translation

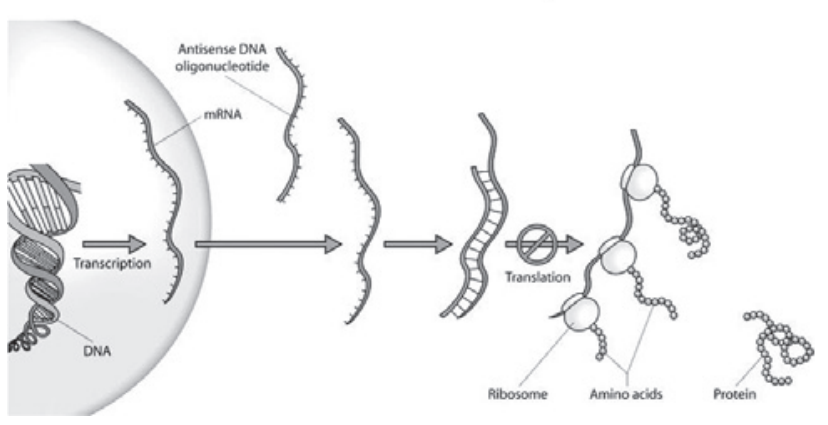

Fig. 2. Schematic showing how antisense DNA strands can interfere with protein translation. This documents the therapy of a genetic disorder. The synthesized antisense compound binds to RNA causing inactivation of the gene that is responsible for the genetic defect. According to Wikipedia. 
Antisense compound can make a cell eliminate a specific exon

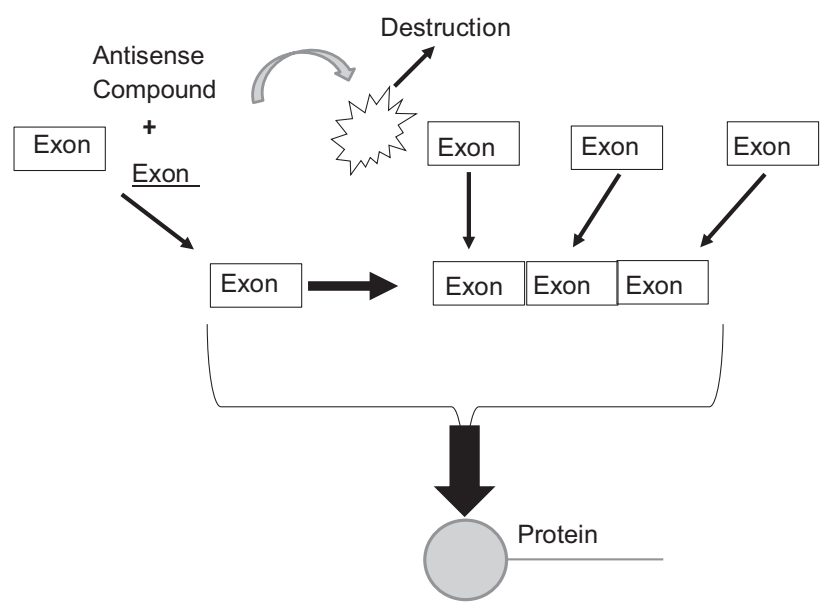

Fig. 3. The regions of genes that carry instructions for proteins are known as exons. Laboratory-designed antisense compounds can make a cell eliminate a specific exon and a functional protein is synthesized from the remaining instructions. According to QUEST Online, Spring 2011 Issue.

RNA produced by that gene and inactivate it, effectively turning that gene "off". This synthesized nucleic acid is termed an "antisense" oligonucleotide because its base sequence is complementary to the gene's messenger RNA, which is called the "sense" sequence. Antisense compounds are designed to have the right nucleotide sequence to bind specifically to and interfere with its associated messenger RNA, the instructions for the production of a particular protein (51) (Fig. 2).

Further research revealed that using antisense technology may be applied to other disorders that affect the exon metabolism, by intervention in the role of exons in controlling the synthesis of proteins. An exon is a nucleic acid sequence that is represented in the mature form of an RNA molecule. Laboratory-designed antisense compounds can make a cell eliminate a specific exon while a functional protein is synthesized from the remaining instructions (Fig. 3 ). Each antisense drug is designed with the right complementary genetic code to bind to a specific sequence of nucleotides in its mRNA target to form a short area of double strands. Thus ISIS 301012 is an antisense oligonucleotide developed to reduce the hepatic synthesis of apolipoprotein B-100. Apolipoprotein B-100 is made in the liver, and antisense oligonucleotides preferentially distribute to that organ, so antisense apolipoprotein B-100 may have a potential as an efficacious lipid-lowering agent (52).

Another antisense compound, APOCIIIRx, is designed to reduce apolipoprotein C-III (apoC-III), a key regulator of serum TG levels. ApoC-III is a 79-amino acid glycoprotein synthesized in the liver that plays a central role in the regulation of serum TGs. Recent data suggest that loss of function mutations within the apoC-III gene lowers the TG levels (53).

Antisense compounds, compositions and methods are provided for modulating the expression of PPARs (peroxisome proliferatoractivated receptors). The compositions comprise antisense com-
Cardiovascular and cancer mortality of males age group 25-64 years

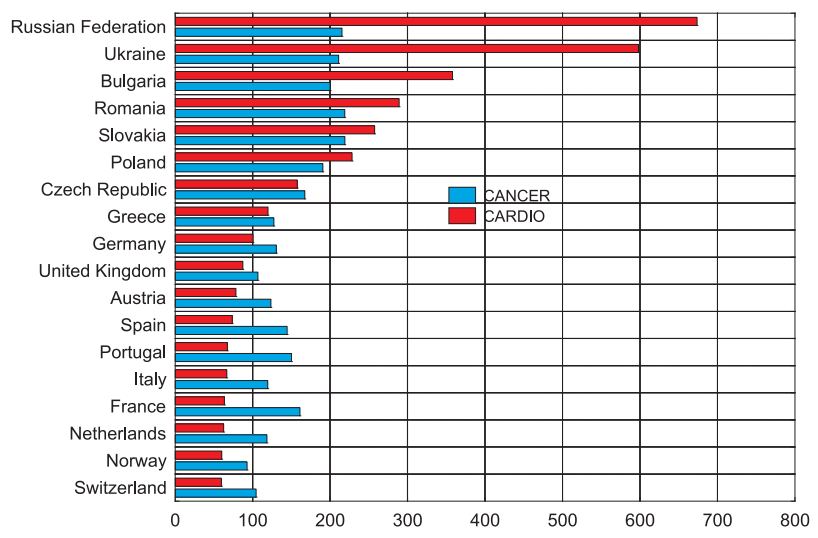

Fig. 4. Cardiovascular and cancer mortality of males in age group 2564 years. According to WHO data (54).

pounds, particularly antisense oligonucleotides, targeted to nucleic acids encoding PPAR-delta. It appears that linking PPARs (Fig. 1) and antisense drugs bears a promise for extensive future use, not only in managing CVD but also for malignancy and other disorders.

Some of these new discoveries may represent a breakthrough in our understanding of disease. Much of this information is very new, being published primarily in patent application prior to undergoing a more lengthy editorial process in scientific journals.

\section{Conclusion}

Cardiovascular disease (CVD) remains a critical component in population mortality. Figure 4 documents that in Eastern Europe, CVD persists as the main cause of mortality in midle age men, substantially exceeding cancer mortality. In Western Europe, malignancy is becoming the foremost cause of mortality (54). Epidemiological data confirmed that regular dietary intake of plant-derived foods (providing antioxidants) and smoking cessation may reduce the risk of CVD and stroke. Another decisive factor leading to a dramatic decline in CVD mortality in recent decades has been the discovery and introduction of effective medications that normalize blood lipids and inhibit vascular inflammation. Credit has to be assigned to a wide use of statins, alone or in combination with niacin and fibrates. In the parts of the world where these medications are accessible, they contributed to lowering of LDL, oxLDL, TG and to optimizing HDL. This review provides an insight into established and novel medications, their mechanism of effect and their potential when used in meaningful combinations.

\section{References}

1. Brinkley RE, Nicklas BJ, Kanaya AM et al. Plasma oxidized LDL levels and arterial stiffness in older adults. Hypertension 2009; 53: 846-852. 
2. Steinberg D. The pathogenesis of atherosclerosis: an interpretive history of the cholesterol controversy, part III: mechanistically defining the role of hyperlipidemia. J Lipid Res 2005; 46: 2037-2051.

3. Hansson GK. Inflammation, atherosclerosis, and coronary artery disease. N Engl J Med 2005; 352 (16): 1685-1695.

4. Das SK, Chakrabarti R. Role of PPAR in cardiovascular diseases. Recent Pat Cardiovasc Drug Discov 2006; 1 (2): 193-209.

5. van Bilsen M, van Nieuwenhoven FA. PPARs as therapeutic targets in cardiovascular disease. Expert Opin Ther Targets 2010; 14 (10): 10291045 .

6. Ferguson JJ. The role of oral antiplatelet agents in atherothrombotic disease. Am J Cardiovasc Drugs 2006; 6: 149-157.

7. N E Barrett, L Holbrook, S Jones et al. Future innovations in antiplatelet therapies. Br J Pharmacol 2008; 154 (5): 918-939.

8. van Heek M, Farley C, Compton DS et al. Ezetimibe selectively inhibits intestinal cholesterol absorption in rodents in the presence and absence of exocrine pancreatic function. Br J Pharmacol 2001; 134 (2): 409-417.

9. Ginter E, Simko V. Statins: the drugs for the 21 st century? Bratisl Lek Listy 2009; 110 (10): 664-668.

10. Prevention of cardiovascular events and death with pravastatin in patients with coronary heart disease and a broad range of initial cholesterol levels. The Long-Term Intervention with Pravastatin in Ischaemic Disease (LIPID) Study Group. N Engl J Med 1998; 339 (19): 1349-1357.

11. Shepherd J, Cobbe SM, Ford I et al. Prevention of coronary heart disease with pravastatin in men with hypercholesterolemia. West of Scotland Coronary Prevention Study Group. N Engl J Med 1995; 333 (20): 1301-1307.

12. Randomised trial of cholesterol lowering in 4444 patients with coronary heart disease: the Scandinavian Simvastatin Survival Study (4S). Lancet 1994; 344 (8934): 1383-1389.

13. Teramoto T, Shimano H, Yokote K, Urashima M. Effects of pitavastatin (LIVALO Tablet) on high density lipoprotein cholesterol (HDL-C) in hypercholesterolemia. J Atheroscler Thromb 2009; 16 (5): 654-661.

14. Ginter E, Simko V. Enigmatic lipoprotein (a) and cardiovascular disease. Bratisl Lek Listy 2010; 111 (10): 570-573.

15. The AIM-HIGH Investigators. The role of niacin in raising high-density lipoprotein cholesterol to reduce cardiovascular events in patients with atherosclerotic cardiovascular disease and optimally treated low-density lipoprotein cholesterol. Am Heart J 2011; 161 (3): 538-543.

16. Cefali EA, Simmons PD, Stanek EJ et al. Improved control of niacininduced flushing using an optimized once-daily, extended-release niacin formulation. Int J Clin Pharmacol Ther 2006; 44: 633-640.

17. Soga T, Kamohara M, Takasaki $\mathbf{J}$ et al. Molecular identification of nicotinic acid receptor. Biochem Biophys Res Commun 2003; 303 (1): 364-369.

18. Duncan RE, Ahmadian M, Jaworski K et al. Regulation of lipolysis in adipocytes. Annu Rev Nutr 2007; 27: 79-101.

19. Frick MH, Elo O, Haapa K et al. Helsinki Heart Study: PrimaryPrevention Trial with Gemfibrozil in Middle-Aged Men with Dyslipidemia. N Engl J Med 1987; 317: 1237-1245.

20. Elam M, Lovato LC, Ginsberg H. Role of fibrates in cardiovascular disease prevention, the ACCORD-Lipid perspective. Curr Opin Lipidol 2011; 22 (1): 55-61.
21. van Bilsen M, van Nieuwenhoven FA. PPARs as therapeutic targets in cardiovascular disease. Expert Opin Ther Targets 2010; 14 (10): 1029-1045.

22. Kuusisto J, Andrulionyte L, Laakso M. Atherosclerosis and cardiovascular risk reduction with PPAR agonists. Curr Atheroscler Rep 2007; 9 (4): 274-280.

23. Petrovski G, Gurusamy N, Das DK. Resveratrol in cardiovascular health and disease. Ann N Y Acad Sci 2011; 1215: 22-33.

24. Wu JM, Hsieh TC. Resveratrol: a cardioprotective substance. Ann N Y Acad Sci 2011; 1215: 16-21.

25. Shekelle PG, Morton SC, Jungvig LK et al. Effect of supplemental vitamin $\mathrm{E}$ for the prevention and treatment of cardiovascular disease. $\mathrm{J}$ Gen Intern Med 2004; 19: 380-389.

26. Saremi A, Arora R. Vitamin E and cardiovascular disease. Am J Ther 2010; 17 (3): e56-65.

27. Riccioni G. Carotenoids and cardiovascular disease. Curr Atheroscler Rep 2009; 11 (6): 434-439.

28. Omenn GS, Goodman GE, Thornquist MD et al. Effects of a combination of beta-carotene and vitamin A on lung cancer and cardiovascular disease. N Engl J Med 1996; 334: 1150-1155.

29. Riccioni G, Mancini B, Di Ilio E et al. Protective effect of lycopene in cardiovascular disease. Eur Rev Med Pharmacol Sci 2008; 12 (3): 183-190.

30. Frikke-Schmidt H, Lykkesfeldt J. Role of marginal vitamin C deficiency in atherogenesis: in vivo models and clinical studies. Basic Clin Pharmacol Toxicol 2009; 104 (6): 419-433.

31. Aguirre R, May JM. Inflammation in the vascular bed: importance of vitamin C. Pharmacol Ther 2008; 119 (1): 96-103.

32. Ginter E. Chronic vitamin $C$ deficiency increases the risk of cardiovascular diseases. Bratisl Lek Listy 2007; 108 (9): 417-421.

33. Ginter E. Vegetarian diets, chronic diseases and longevity. Bratisl Lek Listy 2008; 109 (10): 463-466

34. Crowe FL, Roddam AW, Key TJ et al. Fruit and vegetable intake and mortality from ischaemic heart disease: results from the European Prospective Investigation into Cancer and Nutrition (EPIC)-Heart study. Eur Heart J (2011) doi: 10.1093/eurheartj/ehq465.

35. Riediger ND, Othman RA, Suh M, Moghadasian MH. A systemic review of the roles of n-3 fatty acids in health and disease. J Am Diet Assoc 2009; 109: 668-679.

36. Ginter E, Simko V. Polyunsaturated fatty acids n-3: new data on heart disease, cancer, immune resistance and mental depression. Bratisl Lek Listy 2010; 111 (12): 680-685.

37. Bays HE, Tighe AP, Sadovsky R, Davidson MH. Hypertriglyceridemia is a risk factor for atherosclerotic coronary heart disease. Expert Rev Cardiovasc Ther 2008; 6: 391-409.

38. Massaro M, Scoditti E, Carluccio MA et al. Basic mechanisms behind the effects of n-3 fatty acids on cardiovascular disease. Prostaglandins Leukot Essent Fatty Acids 2008; 79: 109-115.

39. Barter P, Ginsberg HN. Effectiveness of combined statin plus omega-3 fatty acid therapy for mixed dyslipidemia. Am J Cardiol 2008; 102 : 1040-1045.

40. Lioudaki E, Ganotakis ES, Mikhailidis DP. Ezetimibe; more than a low density lipoprotein cholesterol lowering drug? An update after 4 years. Curr Vasc Pharmacol 2011; 9 (1): 62-86. 
41. Taylor AJ, Villnes TC, Stanek EJ et al. Extended- release niacin or ezetimibe and carotid intima- media thickness. N Engl J Med 2009; 361 (22): 2113 .

42. Tenenbaum A, Fisman EZ, Motro M et al. Optimal management of combined dyslipidemia: what have we behind statins monotherapy? Adv Cardiol 2008; 45: 127-153.

43. Reiner Z. Combined therapy in the treatment of dyslipidemia. Fundam Clin Pharmacol. 2010; 24 (1): 19-28.

44. Lin Y, Mousa SS, Elshourbagy $\mathbf{N}$ et al. Current status and future directions in lipid management: emphasizing low-density lipoproteins, high-density lipoproteins, and triglycerides as targets for therapy. Vasc Health Risk Manag 2010; 6: 73-85.

45. Costet P. Molecular pathways and agents for lowering LDL-cholesterol in addition to statins. Pharmacol Ther 2010; 126 (3): 263-278.

46. Hausenloy DJ, Yellon DM. Enhancing cardiovascular disease risk reduction: raising high-density lipoprotein levels. Curr Opin Cardiol 2009; 24 (5): 473-482.

47. Xu DY, Shu J, Huang QY et al. Evaluation of the lipid lowering ability, anti-inflammatory effects and clinical safety of intensive therapy with Zhibitai, a Chinese traditional medicine. Atherosclerosis 2010; 211 (1): 237-241.

48. Rosenson RS. Lp-PLA (2) and risk of atherosclerotic vascular disease. Lancet 2010; 375: 1498-1500.

49. Barrett NE, Holbrook L, Jones $S$ et al. Future innovations in antiplatelet therapies. Br J Pharmacol 2008; 154 (5): 918-939

50. Mizuno Y, Jacob RF, Mason RP. Inflammation and the development of atherosclerosis: effects of lipid-lowering therapy. J Atheroscler Thromb 2011; 18: 000-000 (in press).

51. Yamamoto T, Nakatani M, Narukawa K, Obika S. Antisense drug discovery and development. Future Med Chem 2011; 3 (3): 339-365.

52. Akdim F, Stroes ES, Kastelein JJ. Antisense apolipoprotein B therapy: where do we stand? Curr Opin Lipidol 2007; 18 (4): 397-400.

53. Ooi EM, Barrett PH, Chan DC, Watts GF. Apolipoprotein C-III: understanding an emerging cardiovascular risk factor. Clin Sci (Lond) 2008; 114 (10): 611-624.

54. European Health for All database (HFA-MDB). Copenhagen, WHO Regional Office for Europe, (2011) 